Compar at i ve st udi es on super soni $\mathrm{c}$ free $\mathrm{j}$ et $\mathrm{s}$ from nozzl es of compl ex geometry

\begin{tabular}{|l|l|}
\hline 著者 & $\begin{array}{l}\text { RAO Sr i sha M V., ASANO Shi ngo, SAl TO } \\
\text { Tsut omi }\end{array}$ \\
\hline $\begin{array}{l}\text { j our nal or } \\
\text { publ i cat i on t i t l e }\end{array}$ & Appl i ed Ther mal Engi neer i ng \\
\hline vol une & 99 \\
\hline page r ange & $599-612$ \\
\hline year & $2016-04$ 25 \\
\hline URL & ht t p: //hdl . handl e. net /10258/00008912 \\
\hline
\end{tabular}


Compar at i ve st udi es on super soni $\mathrm{c}$ free $\mathrm{j}$ et $\mathrm{s}$ from nozzl es of compl ex geometry

\begin{tabular}{|l|l|}
\hline 著者 & RAO Sr i sha M V., ASANO Shi ngo, SAl TO Tsut oma \\
\hline $\begin{array}{l}\text { j our nal or } \\
\text { publ i cat } \mathrm{i} \text { on } \mathrm{t} \text { i t l e }\end{array}$ & Appl i ed Ther nal Engi neer i ng \\
\hline vol une & 99 \\
\hline page r ange & $599-612$ \\
\hline year & 2016- O4 25 \\
\hline URL & ht t p: //hdl . handl e. net /10258/00008912 \\
\hline
\end{tabular}




\title{
Comparative studies on supersonic free jets from nozzles of complex geometry
}

\author{
Srisha M.V. Rao, Shingo Asano, Tsutomu Saito \\ Department of Aerospace Engineering, \\ Muroran Institute of Technology, Muroran City, Hokkaido, Japan 050-8585.
}

\begin{abstract}
Experimental and numerical studies are conducted to compare free jets from different supersonic nozzles for mixing enhancement. A conical nozzle of Mach 1.80 is the reference. Three complex nozzles are a beveled nozzle, a nozzle with six chevrons and a six lobed ESTS nozzle [1]. All nozzles have the same throat diameter and designed average exit Mach number. The studies are conducted at $\mathrm{NPR}=6$, using acetone PLIF, centerline pitot pressure measurements and 3D RANS simulations. A novel method of decomposing PLIF images based on intensity histogram and then recomposing after applying selective gains to emphasize the growth of shear layers is discussed. PLIF images are processed to extract the growth rate of jet width which indicates the rate of mixing. The ESTS lobed nozzle shows the highest enhancement of mixing (430\%) followed by chevron (222\%) and the beveled nozzle with a moderate (138\%). The numerical simulations are validated and agree well with experimental results. ESTS lobed nozzle is found to produce widespread streamwise vortices compared to clustered vortices of the chevron nozzle. The mechanism of streamwise vorticity production from ESTS nozzle is clarified for the first time in this study.
\end{abstract}

Keywords: Mixing Enhancement, Supersonic Shear Layers, Supersonic Jets, Complex Nozzles

Email addresses: srisha.raomv@gmail.com (Srisha M.V. Rao), 14042003@mmm.muroran-it.ac.jp (Shingo Asano), saito@mmm.muroran-it.ac.jp (Tsutomu Saito) 


\section{Introduction}

Novel supersonic nozzles with complex exit geometries are excellent passive techniques to enhance jet mixing rate $[1,2,3,4]$. Enhancement of mixing at supersonic speeds becomes particularly important after extensive research has conclusively shown that compressibility effects reduce growth rate of mixing layers $[5,6]$. Several engineering applications such as supersonic ejector, high speed airbreathing engines (SCRamjet engines) and aeroacoustic noise features of jet exhausts are solely dependent on the characteristics of mixing at supersonic speeds. A wide array of supersonic mixing enhancement techniques was reviewed by Gutmark et.al. [7], and the effectiveness of passive techniques that use geometrical modifications at the trailing edge of the nozzle was described. Lobes proceeding from the throat to exit of the nozzle that alternately deeply penetrate the core supersonic flow at the lobe trough and expand at the lobe crest are the characteristic of lobed supersonic nozzles [4, 3]. Large streamwise vorticity generated by such convoluted nozzle structure was shown to have increased the mixing and entrainment rate greatly. They were particularly useful for applications in supersonic ejectors [4, 8] and for improvements in supersonic combustion [9]. However, large stagnation pressure loss due to complex shock structure associated with such nozzles was also reported [8]. Recently, efforts have been made to optimize the lobe geometry to maximize pressure recovery in supersonic ejector [10]. Tabs, on the other hand, were discrete simple triangular shaped protrusions placed at the nozzle exit with only moderate penetration into the jet core flow [11]. Number of studies using optical tools have shown the vortex evolution from such tabs that lead to enhanced mixing in jets $[12,13,14]$. Chevron nozzles evolved from tabbed nozzles with an aim to reduce parasitic stagnation pressure loss as detailed in the review by Zaman et.al. [15]. Chevrons are continuous sawtooth shaped modification to the exit of the nozzle with minimal penetration into the jet core flow in comparison to the tabs [15] that have now found practical use in mixing enhancement and noise reduction from jet exhausts $[16,17,18,19]$. Recently, Kong et.al. [2] have shown an increase of $14.8 \%$ of entrainment ratio when chevron nozzles were used within the supersonic ejector. A simple modification to the round supersonic nozzle is by beveling it at a certain angle thereby upsetting its symmetry which has shown a deflection of the jet towards the shorter end of the bevel, an increase of mixing and noise reduction in various studies [20, 21]. 
There are many modifications to these template shapes that have been studied by different investigators such as the clover nozzle [22] and cross-shaped nozzle [23] (lobed nozzles); or crown nozzle [24] (chevron nozzle).

- A novel lobed nozzle was devised by Rao and Jagadeesh [1], after giving due consideration to the fact that deep penetration and extreme convolution of the geometry caused severe stagnation pressure losses in the lobed nozzles. The new nozzle named as ESTS(Elliptic Sharp Tipped Shallow) lobed nozzle has the advantage that it is easy to produce (being formed by angular drilling from location offset from the center of the nozzle) and has shallow penetration thereby reducing stagnation pressure losses considerably. The free jet flow structure from a four lobed ESTS nozzle was explored using the Laser scattering method and application within a supersonic ejector showed significant improvement of entrainment ratio by $30 \%$.

. Specification of complex nozzles involve combination of multiple geometrical parameters, such as number of lobes/chevrons, length/width/depth of lobes/chevrons and penetration angles to list a few. Further, it has been observed that small changes to certain parameters can bring about drastic changes to the flow phenomena in a non-linear manner [15, 17], making optimization a very laborious task yet to be fully accomplished [10]. This complicates the understanding of such nozzles towards a cohesive design framework since the domain of parametric combinations is very large. Most of the studies try to differentiate the flow mechanism of one kind of nozzle with a reference round nozzle. Comparative studies among nozzles of different kinds are few, limited to comparisons in particular applications such as noise reduction or mixing enhancement in supersonic ejectors $[18,13,23]$. Though, eventually all the complex nozzles aim to produce large vorticity, there are fundamental differences in the manner of their production. The lobed nozzles involve an azimuthal variation of Mach number within the core supersonic flow right from the nozzle throat to the exit of the nozzle. In contrast, the chevrons are introduced only very close to the exit of the nozzle and the supersonic flow in a large part of the nozzle is essentially not very different from the round conical nozzle. This is bound to produce differences in vorticity production and hence the rate of mixing. These observations motivate this study of comparing the supersonic free jet flow from four supersonic nozzles belonging 
to different classes of geometrical modifications.

. The supersonic free jet is an ideal platform to fundamentally study such aspects since it offers much greater optical access compared to confined jets. Being easy to set up, much of the previous work also have been carried out on supersonic free jets. Laser based optical tools can easily slice through different sections of this complex three dimensional flow field giving a detailed comparison among different nozzles. In this study we utilize the Planar Laser Induced Fluorescence (PLIF) technique using acetone as the seeding agent within the supersonic jet. Though Mie scattering of laser from seeded particles produces strong signals that are readily visualized through digital cameras, it is difficult to distinguish between seeded and entrained particles when such interactions occur. PLIF is sensitive only to that particular seeding particle that can produce florescent emissions upon excitation from a definite wavelength of light. This is an advantage when conducting experiments to study mixing since the tagging of the flow and spread of the passive tracers is unambiguously captured. Acetone PLIF has been applied in the study of supersonic free jets $[25,26]$ and supersonic flows in tunnels as well $[27]$.

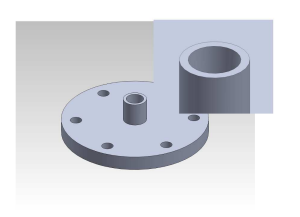

Type A

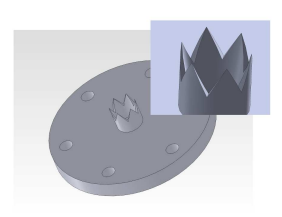

Type C

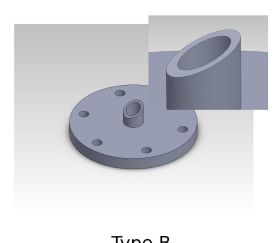

Type B

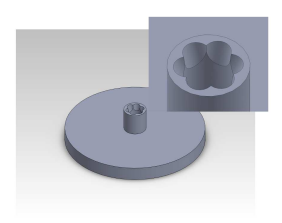

Type D

Figure 1: The four different nozzles tested in this study. The insets show details of the nozzle contour. Type A is the reference conical nozzle. Type B is a bevel nozzle where a bevel of $26^{\circ}$ is introduced on the reference geometry. Type $\mathrm{C}$ has chevrons cut at the exit of the reference geometry. Type D is an ESTS lobed nozzle with the average exit Mach number same as the reference nozzle. 
. The objective of this current study is to compare the supersonic free jet structures from four different nozzles which are shown in Figure 1. Type A is Mach 1.80 conical supersonic nozzle with round exit and is the reference. Type B is the simplest modification to the nozzle geometry wherein the geometry of Type A is beveled off at $26^{\circ}$. Six chevrons are cut at the exit of the reference nozzle to form the chevron nozzle Type C. Type D is an ESTS lobed nozzle with six lobes designed such that the exit area is the same as that of Type A and hence the average exit Mach number is 1.80 [1]. The nozzles shall henceforth be referred to as Type A, Type B, Type C and Type D respectively. The experimental study involves the use of acetone PLIF to capture flow visualizations along the streamwise and spanwise planes. Pitot measurements are carried out along the centerline which indicate the rate of change of centerline Mach number. To fully understand the details of the complex three dimensional flow field numerical studies are conducted using FASTAR an unstructured CFD code developed by JAXA, Japan. Full three dimensional RANS equations are solved using the Spalart-Allmara's turbulence model. The numerical results are in good agreement with the experimental visualizations as well as the centerline pitot measurements. Results that compare and contrast the flow features from the four different nozzles are described in this article. The rate of mixing is evaluated from acetone PLIF images by extracting the rate of growth of jet width. A new methodology to process the acetone PLIF images by decomposing the images according histogram of intensities is described that enables to emphasize the growth of mixing layers in the images. Numerical results are used to elaborate on mechanisms for increase in mixing through vorticity distributions. The mechanism of vorticity production from ESTS lobed nozzle is clarified for the first time.

. The details of the experimental setup and diagnostics used are first described. This is followed by a description of the analysis of experimental data, particularly the processing of acetone PLIF images. Details of the numerical procedure are given next. A discussion where the results from experiments and numerical simulations are used to compare the four supersonic nozzles is detailed before concluding the article. 


\section{Experimental Facility}

The experiments are carried out on a jet facility which has arrangements to seed acetone in the supersonic jet and appropriate optical arrangements to produce laser sheet for PLIF as schematically shown in Figure 2. A regulated supply of high pressure air is delivered to the stagnation chamber (40 $\mathrm{mm}$ in diameter, 90 $\mathrm{mm}$ in length) from gas bottles. The compressed air is bubbled through an acetone seeder unit before it is stagnated in the stagnation chamber. The supersonic nozzles are mounted interchangeably at the downstream end of the stagnation chamber unit. The stagnation pressure of the jet is measured at the stagnation chamber using a KYOWA pressure indicator. The blow down operation is controlled by a solenoid valve and the pressure is set at the pressure regulator. The jet exits to the ambient and an exhaust system with a wide hood is provided far downstream of the jet to purge the air-acetone mixture. The diagnostics used are PLIF for flow visualizations and centerline pitot measurements. In all further discussion, the center point of the nozzle exit is considered the geometrical origin and the $\mathrm{Z}$ axis refers to the streamwise direction. The $\mathrm{X}-\mathrm{Y}$ planes form the cross-sectional planes.
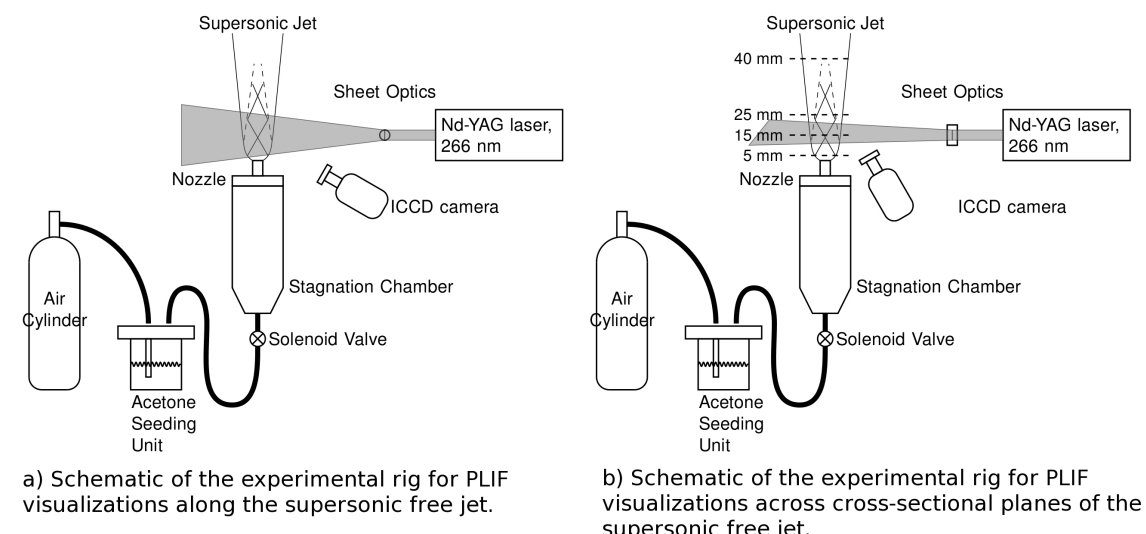
supersonic free jet.

Figure 2: Schematic illustration of the experimental arrangement. a) shows the optical arrangement when PLIF images are taken along the streamwise planes and b) shows the setup for cross-sectional imaging where the camera is placed just behind the exit of the nozzle. 


\subsection{Geometrical Details of Supersonic Nozzles}

Type A is a standard conical supersonic nozzle with a throat diameter of 5 $\mathrm{mm}$ and an exit of $6 \mathrm{~mm}$ diameter such that the expected Mach number based on ideal isentropic area relations is 1.80 . Type $\mathrm{B}$ is produced by beveling the geometry of Type A such that the angle of bevel is $26^{\circ}$ to the $\mathrm{X}-\mathrm{Y}$ plane. Type $\mathrm{C}$ takes the same geometry as Type A and six isosceles shaped chevrons are cut at the exit of the nozzle. The manner of design of Type B and Type C nozzles are according to Tide and Srinivasan [18]. Type D is a six lobed ESTS lobed nozzle produced using the methodology describe in Rao and Jagadeesh [1] such that the throat area is $5 \mathrm{~mm}$ and exit area is the same as Type A. Throat diameter is taken as the reference dimension $\mathrm{D}$ which is the same for all the nozzles.

\subsection{Acetone Planar Laser Induced Florescence (PLIF) setup}

The optical arrangement is similar to the setup used in Hatanaka et.al. [26] and is described briefly here. Acetone has been found to be a useful seeding agent for PLIF technique due to its relatively high florescence characteristics, low toxicity and high vapor pressure which makes seeding easy by bubbling [28]. The fourth harmonic (UV - $266 \mathrm{~nm}$ ) of a Q-switched Nd-YAG laser system (pulse width $4 \sim 6 \mathrm{~ns}$, energy $80 \mathrm{~mJ} /$ pulse and pulse rate $10 \mathrm{~Hz}$ ) is rendered by sheet optics into a sheet about $30 \mathrm{~mm}$ in height and less than $0.5 \mathrm{~mm}$ in width at the nozzle exit. Seeded acetone molecules from the jet are excited to higher energy levels by the laser and florescence at the blue end of the visual spectrum is captured by the CCD camera (Hamamatsu, C8484-05C, 1024x1024 pixels). The florescence signals are rather weak and image intensification was done using (Hamamatsu, M7971-51). A band-pass optical filter (Schott Inc, BG12) was used to filter in only florescence emission. The camera and the laser are synchronized using a pulse generator (Stanford Instruments DG535). While the rest of the optical system remained the same the laser sheet orientation and camera placement were changed according to whether streamwise planes or cross sectional planes were being captured. During streamwise plane visualizations, the camera is placed normal to the laser sheet that cuts along the length of the jet as shown in Figure 2a. At the resolution of $1024 \times 1024$ pixels, the image has a scale of $0.035 \mathrm{~mm} /$ pixel which is the same in both $\mathrm{X}$ and $\mathrm{Z}$ directions. The image covers an extent of $6 \mathrm{D}$ from the exit of the nozzle. The laser sheet is passed a short 
distance above the nozzle lip to avoid strong reflections from the metallic surface of the nozzle. For cross-sectional visualizations, the laser sheet is made to cut along the $\mathrm{X}-\mathrm{Y}$ planes at different $\mathrm{Z}$ locations, while the camera is placed just upstream of the nozzle exit viewing the planes at a perspective angle (Figure $2 b)$. The camera focus remains fixed during the streamwise visualizations but is varied from section to section during cross-sectional visualizations. During a particular run 30 images are captured during the test time and five such runs are conducted to get number of images for ensemble averaging.

\subsection{Centerline pitot measurements}

Pitot measurements are carried out along the axis at discrete $\mathrm{Z}$ locations (ranging from 4D to 20D) downstream of the jet exit. The pitot is not placed very close to the nozzle exit to avoid blockage effects that can potentially cause large disruptions to the supersonic flow from the nozzle. A cylindrical pitot of 0.9 $\mathrm{mm}$ diameter is used and is connected to a KYOWA piezoelectric pressure sensor ( $\pm 1 \%$ accuracy). Pressure signals are acquired at a sampling rate of $5 \mathrm{kHz}$ and are later digitally processed to extract the mean pitot pressures at the locations.

\subsection{Experimental Conditions}

The experiments are carried out such that the average Nozzle Pressure Ratio (NPR, ratio of stagnation pressure of jet to the ambient pressure) is maintained at 6 at all times. This condition corresponds to the NPR for correct expansion in the reference nozzle Type A. The average stagnation pressure measured in the chamber is 6.07 bar $( \pm 4 \%)$ and the average ambient pressure measured is 1.0123 bar $( \pm 2 \%)$ giving a NPR of 5.99. Typical blow down time is $5 \mathrm{~s}$ and a steady test time of about $2 \sim 3 \mathrm{~s}$ is achieved. For every nozzle case 5 runs are conducted to ensure statical consistency.

\section{Data Analysis}

\subsection{Analysis of PLIF images}

The intensity of acetone florescence emission is primarily dependent on the distribution of number density of acetone molecules [26, 29]. A detailed multistep decay model of acetone florescence shows that the flouescence intensity is also affected by pressure, temperature and presence of oxygen which acts as a 
quenching agent $[30,31,32]$. The effect of oxygen quenching is clearly evident in the comparison of acetone PLIF images of supersonic jets using nitrogen and air [25]. Thus, the intensity of acetone PLIF is dependent on multiple parameters and depending on which of the different parameters is maintained constant the acetone PLIF signal can be used for measuring different parameters such as concentration or temperature as the case maybe [33]. But, supersonic flow contains regions of large pressure, temperature and concentration gradients where all the effects of multiple parameters become important. Even in largely shock free supersonic flows the effects of pressure and temperature has to be considered explicitly, if not they introduce uncertainty in quantitative data analysis [27]. The supersonic jet exiting into ambient is a shock containing flow and hence the temperature and pressure distribution also have to be known to undertake precise quantitative analysis of intensity data which is difficult. However, the objective of the current work is to compare the spreading rates of jets from different geometries. Given that the presence of acetone is registered as intensity signals in the PLIF image, the extent of jet spread can be easily extracted from PLIF images. In the process of extracting the information of width of jet spread from streamwise PLIF images, a unique method to decompose the images according to the histogram of intensity distribution was developed which emphasizes the growth of mixing layers in the jet. This procedure is described in detail as follows.

\subsubsection{Image decomposition according to histogram levels}

Before capturing PLIF images with the jet turned on, background images are captured with the laser on and at no flow conditions. The first step of image processing is to remove any ambient background noise by subtracting the average no flow background image. The state of images at each step of processing is clearly shown in Figure 3. The images shown are averaged over 30 images per set for clarity in visibility of flow features. Acetone PLIF signals are rather weak and it becomes necessary to average the images to obtain an image where flow features can be interpreted clearly [25]. The image processing techniques are applied to individual images before averaging. The image processing procedure is discussed by considering a particular case of Type A nozzle and the results pertaining to different nozzles are discussed in Section 5.1. A look at the background subtracted image clearly shows the flow features such as shocks in the core of the jet, but 


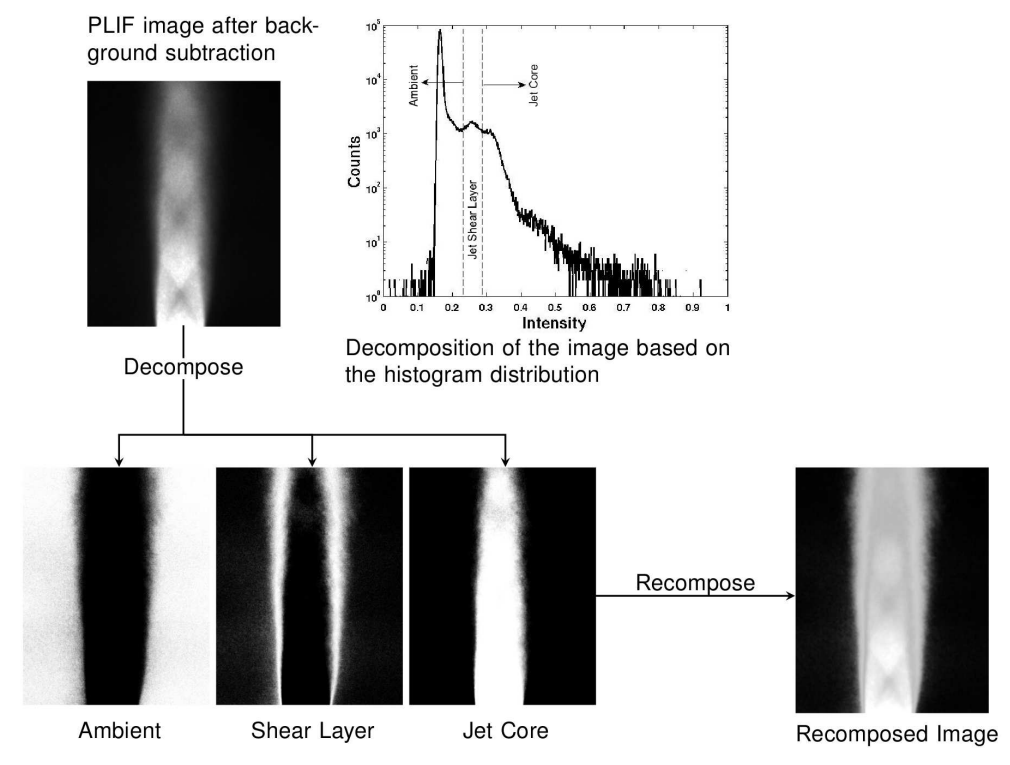

Figure 3: Step by step procedure of PLIF image processing showing image decomposition by histogram and then recomposition by applying selective gains to emphasize the mixing layer in PLIF image.

the jet spread is not readily visible because the intensity levels contained in the jet shear layer where acetone containing jet mixes with the ambient is less in contrast to the jet core. The intensity of acetone PLIF is primarily a function of acetone concentration distribution. The highest concentrations of acetone are found along the jet core and the concentration decreases across the jet shear layer and is negligible in the ambient. Therefore it is important to emphasize the mixing layer in the PLIF images. Considering that the image is of $1024 \times 1024$ pixels, and the jet occupies only the central portion of the image and the rest are regions of the ambient. The maximum number of pixels are associated with very low values of intensity as seen in the histogram image. The intensity $\left(\mathrm{I}_{\text {mode }}\right)$ associated with maximum pixel counts lies within the ambient region. Thereafter the image is decomposed into regions containing intensity within a certain range. Three such regions are identified within the image according to the intensity ranges defined in Table 1. These range of parameters are found through visual identification from a select number of images and then applied throughout for all other images in the analysis. Thus, this particular procedure is general. However, 
the identification of the intensity ranges is specific to the flow dynamics being studied and the optical arrangements. A new set of intensity ranges may have to be defined when using this procedure on similar problems.

$\begin{array}{ll}\text { Ambient } & \mathrm{I} \leq 1.25 \mathrm{I}_{\text {mode }} \\ \text { Jet Shear Layer } & 1.25 \mathrm{I}_{\text {mode }}>\mathrm{I} \leq 1.65 \mathrm{I}_{\text {mode }} \\ \text { Jet Core } & \mathrm{I}>1.65 \mathrm{I}_{\text {mode }}\end{array}$

Table 1: Definition of the intensity ranges for each decomposition of the PLIF image signals.

. The primary aim of this procedure is to enhance the intensity levels found in the mid range of intensity according to the distribution given in Table 1. However, in this particular case of free jets it is possible to associate a physical flow feature corresponding to the intensities found in different regions. The regions corresponding to these intensity ranges are shown in the histogram figure and also shown split into three distinct regions Ambient, Jet Shear Layer and Jet Core. Clearly the central intensity region lies in the jet shear layer. The next step is to apply different gains to the different regions so that the real jet spread becomes distinctly visible. The gains applied are $\mathrm{G}_{\text {ambient }}=0.25, \mathrm{G}_{\text {shearlayer }}=1.25$ and $\mathrm{G}_{\text {jetcore }}=1.15$ respectively. Thus, the intensity levels in the jet shear layer are amplified more than the jet core and intensity levels in the ambient are suppressed. The image is recomposed after applying these gains to corresponding regions and the recomposed image is seen where jet spread is dominantly emphasized. These steps are important since they clearly bring out the extent of jet spread along $\mathrm{Z}$ axis in the acetone PLIF images. Once the recomposed images are obtained, then the variation of jet boundary width along the $\mathrm{Z}$ axis can be easily calculated from the images [1] which is a significant metric to evaluate the rate of jet mixing with the ambient. The average growth rate of the jet boundary which is the average slope of the line representing jet boundary width vs $\mathrm{Z}$ is directly proportional to the rate of mixing in the jet and this value can be used to compare the different nozzles which described in detail in Section 5.1. It is important to emphasize here that this procedure is designed to make the PLIF images show up the extent of jet spread based on relative intensity levels. A true calibration of acetone PLIF signals is much more involved and complexities of the multi-parameter nature of acetone florescence has to be considered in detail which has not been attempted here. The large variation in acetone florescence 
intensity near shocks is due to significant effects of pressure, temperature and quenching.

\subsubsection{Area of jet spread in cross sectional images}

Another metric that is evaluated for comparison among the four nozzles is the area of jet spread in cross-sectional images. This is done by considering all the pixels that are contained in the region where intensity $\mathrm{I} \geq 0.2 \mathrm{I}_{\max }$ in the image, where $I_{\max }$ is the maximum intensity that is usually found at the center of the PLIF image. The area of all such pixels is computed. Care is to be taken that images at a particular $\mathrm{Z}$ location are only compared since the camera perspective angle is not constant when $\mathrm{Z}$ location is changed. The results are described in Section 5.2.

\subsection{Pitot pressure}

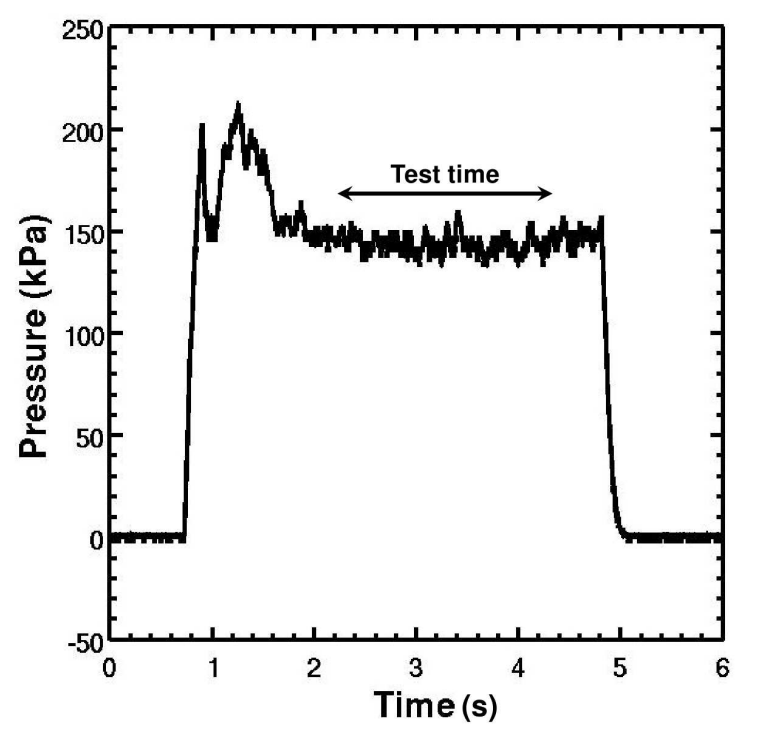

Figure 4: Typical pressure signal measured during centerline pitot pressure measurement.

A typical pressure signal obtained during the pitot pressure measurement along the centerline is shown in Figure 4. The initial rise is due to the sudden opening of the solenoid valve that soon settles down to a constant steady mean pressure that lasts about $3 \mathrm{~s}$ as marked in the figure. An average of over a 
second duration is taken as the mean steady pressure. The fluctuations are small less than $3 \%$ of the mean value. The reported values are an ensemble average of five test runs for every measurement point. The deviation in the ensemble averaged mean pressure is less than $2 \%$, where as the expected uncertainty is $6.5 \%$ considering all factors (Section 3.3), which shows the statistical consistency and reliability of the experiments and measurement technique.

\subsection{Uncertainty Analysis}

An uncertainty of $\pm 4 \%$ exists for stagnation pressure measurements including accuracy of the sensor $( \pm 1 \%)$ and operation of mechanical elements. The atmospheric pressure is known within $\pm 2 \%$ and hence the Nozzle Pressure Ratio is determined with an uncertainty of $\pm 5 \%$. Pitot pressure measurement is intrusive and uses a finite area tube which introduces errors besides the accuracy of the sensor $( \pm 1 \%)$ such as expected variation across the pitot area $( \pm 3 \%)$, effect of viscosity $( \pm 2 \%)[34]$. The pitot pressure measurement has an expected uncertainty of $( \pm 6.5 \%)$ considering the cumulative effects of other sources of experimental error. Image processing algorithms used in this work are primarily concerned with linear measurements $( \pm 3 \%)$ from images with procedures to enhance intensity accordingly. The average jet width evaluated from the experiments is known within $\pm 6 \%$ accounting for the cascading effects of related uncertainty and similarly the average jet width growth rate is evaluated to within $\pm 9 \%$ uncertainty.

\section{Numerical Analysis}

Numerical Computations are carried out using FASTAR, an unstructured CFD code developed by JAXA, Japan. Details of the code are described by Hashimoto et.al. [35] and FASTAR has been validated in simple as well as complex turbulent flow scenarios [36]. The numerical method is briefly described here and the grid and boundary conditions are discussed. The three dimensional Reynolds Averaged Navier Stokes (RANS) equations are solved using a cell centered finite volume scheme. Ideal gas law is applied and the Sutherland's law is used to calculate viscosity. The code uses MUSCL type linear reconstruction and AUSM+ scheme with minmod limiter is used to compute the numerical fluxes. Gradients are evaluated using the Green Gauss method. Time-integration is carried out using LU-SGS (Lower/Upper Symmetric Gauss Seidel) yielding second 
order accurate solutions both in space and time. The computations are carried out on a cluster system developed in-house using PCs. Intel's Core i7-3930K (3.20 $\mathrm{GHz}$ ) processors are used in the cluster that has $24(\mathrm{PE})$ computing elements with a memory of 2.67 GB per element.

. The Reynolds number (based on the reference diameter) at the nozzle throat which remains same in all the cases is $4.376 \times 10^{5}$ and considering nozzle Type A, the exit Reynolds number is $4.465 \times 10^{5}$, implying that the flow is turbulent. Thus, an appropriate turbulence model has to be used to evaluate the effects of turbulent stresses in the RANS equations [37]. The Spalart-Allmara's (SA) one equation turbulence model has been found to be effective with low computational costs. The SA model has been applied to study of supersonic flows in general [38] and to jet flows in particular, including simulations of the screech tones $[39,40]$. Therefore, the Spalart-Allmara's one equation model is used in the present simulations.

\subsection{Grid and Boundary Conditions}

The cylindrical computational volume includes the stagnation chamber, the supersonic nozzle and an ambient that is made large enough such that the boundary effects are negligible. The ambient extends to 100D around the jet and to a distance of $200 \mathrm{D}$ in the $\mathrm{Z}$ direction. Figure 5 shows the grid for Type D, an ESTS supersonic lobed nozzle having complex exit geometry. The grid is made of hexahedral elements which are very fine near the nozzle and coarsened gradually to the periphery as shown in the figure. The insets show the fine nature of the grid near the nozzle exit which is the region of interest. The cell size is nearly a cube of about $0.1 \mathrm{~mm}$ in this region and the closest cell near the wall is 10 $\mu \mathrm{m}$ away from it. The cross-sectional figure shows that the complex geometry has been captured finely by means of the hexahedral mesh. Similar fine quality meshes are generated for all the nozzles. Studies have been conducted using 4, 6 and 8 million cells respectively and the grid converged solution at the finest mesh of 8 million is reported here. The computational method as well as the grid are able to capture the fluid dynamics of the jets and compare well with the experiments as described in Section 5.3. 


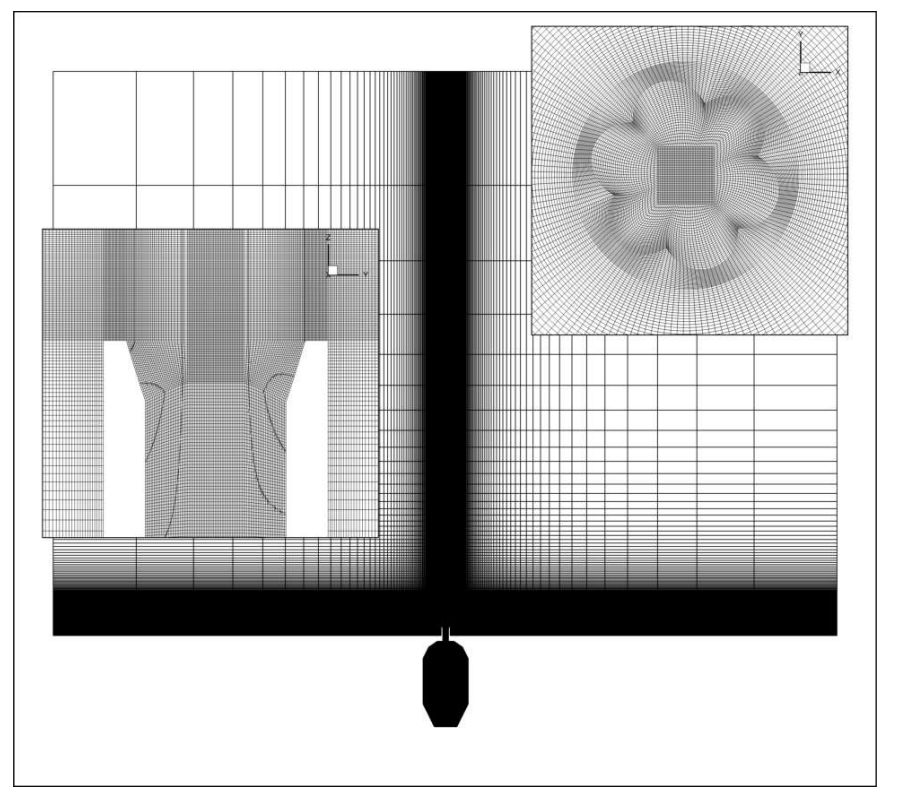

Figure 5: The computational grid (8 million cells) for Type D nozzle showing the overall computational domain and the insets showing the fine grid near the nozzle.

. The stagnation pressure of the jet is imposed at the inlet boundary to the stagnation chamber, while the ambient pressure is prescribed on the boundaries. The walls of the nozzle and stagnation chamber are considered adiabatic and no-slip boundary condition is applied. The simulations are marched in time to steady states. The residuals are converged to the order of $10^{-9}$.

\section{Results and Discussions}

\subsection{Streamwise Acetone PLIF}

Figure 6a compares the average PLIF images taken along the streamwise planes for four different nozzles. For Type C, the laser sheet is made to pass along locations that connect diametrically opposite tips and gaps in the chevron structure. Similarly for Type D the laser sheet is made to pass along the lobe crests and troughs (which is sharply tipped in case of the ESTS nozzle [1] and hence referred as the lobe tip). The cut sections of nozzles according to the respective locations of the laser sheet are placed below each of the PLIF images to demonstrate the locations of the laser sheet and are not at the same scale of 


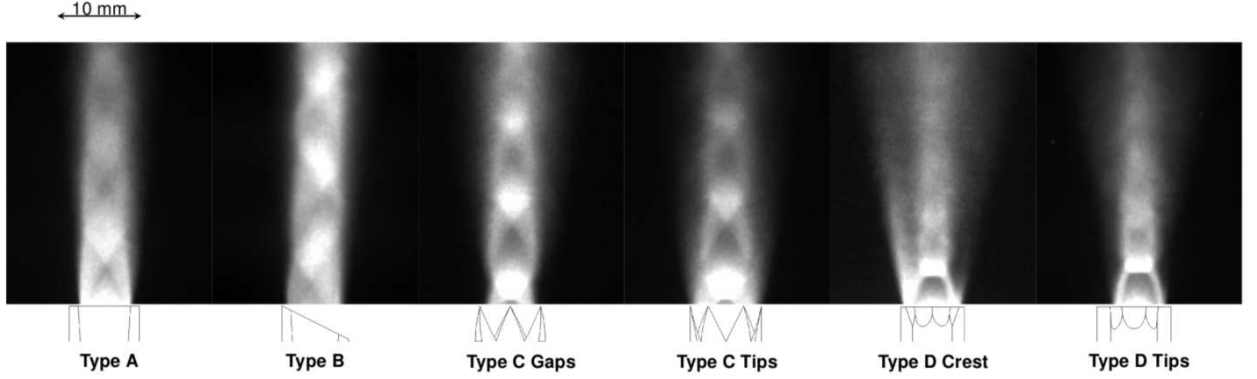

a) Average acetone PLIF images for Type A,B,C and D nozzles.

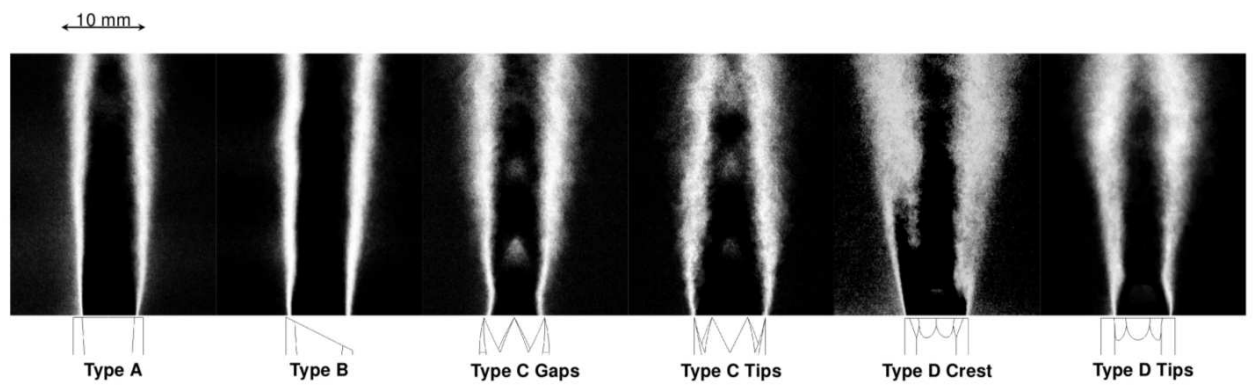

b) Images corresponding to the central region of image decomposition based on the intensity histogram termed as the Jet Shear Layer since the range of intensities emphasize the mixing layer extent in the jet.

$$
\stackrel{10 \mathrm{~mm}}{\longrightarrow}
$$

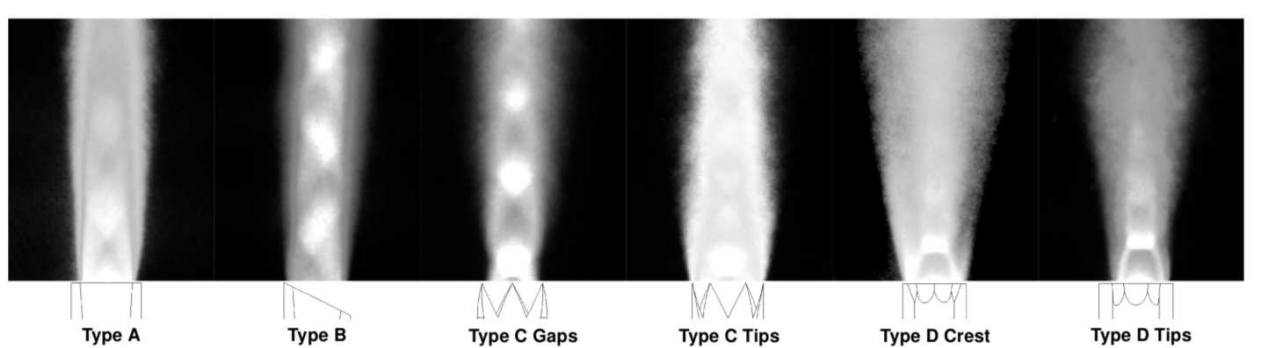

c) Recomposed images of the free jet from the supersonic nozzle which shows the jet width clearly.

Figure 6: Acetone PLIF images along streamwise planes of the different nozzles. Each image is an average of 30 images. The shear layer enhanced images and recomposed images after application of selective gains are also shown. 
the PLIF images. The scale of the PLIF images which is the same for all the nozzles is indicated at the top left of the figure.

. The jet NPR is close to the correctly expanded condition of nozzle Type A thus the jet shock structure consists of oblique shocks. Strong Mach disks that are seen in severly underexpanded jets or shock structures emanating within the nozzle as observed in overexpanded jets are absent. The average PLIF image shows a nearly axi-symmetric configuration with small jet spreading in the observation window (6D). The jet core near the nozzle exit contains the highest concentrations of acetone hence is visible brightly. The concentration of acetone in the jet core decreases gradually in the $\mathrm{Z}$ direction due to mixing with ambient air and thus the intensity of PLIF can be seen to reduce along the $\mathrm{Z}$ axis downstream of the jet exit. The jet mixes with the ambient along the jet mixing layers and the acetone intensity decreases away from the jet in the radial direction also. This change of intensity to very small ambient values in the mixing layer along the radial direction is not immediately apparent in the images because of the strong contrast of the high intensity jet core. It is to make this clear that the image processing steps described in 3.1.1 are executed on the PLIF images.

. The break of symmetry in Type B nozzle is clearly evident by very slight bend of the jet towards the lower end of the bevel and the zig-zag nature of the shock structure. The shock structure is seen to converge close to the higher end of the bevel first and then in the next shock cell it is seen to move close to the lower end. However, besides these changes, the jet structure does not seem to be modified to a great extent.

- The jet structure undergoes significant changes in the PLIF images seen for Type C. A shock cell is seen to emerge from within the chevron structures, converging slightly above the exit plane of the nozzle and then continuing further as shock cells. The first shock cell appears to be a short Mach disk implying that for a very short region small pocket of subsonic flow might be present. What is more significant is that the mixing layer regions contain higher intensity levels (especially as seen in the PLIF image at the tips of Type C) and are thicker than that for Type A. This clearly implies enhanced levels of mixing. 
. Clearly, the flow structure in Type D is drastically different from Type A. The very rapid increase of the jet width and significant intensities in the mixing region is evident in both the images along the crests and the tips. A Mach disk with different widths along the crest and the tips showing its three dimensional nature is evident. The presence of such a wide Mach disk ensures significant subsonic pocket. At least one more shock cell is clearly visible, however, further downstream the absence of shock structures indicate subsonic flows. Thus the nozzle is able to achieve subsonic velocities at the centerline far quicker than any of the other nozzles and the rapid increase in mixing is evident.

- The average images corresponding to those regions in the PLIF image that are contained in the mid-region of the intensity histogram as described in Section 3.1.1 are compared in Figure $6 \mathrm{~b}$ for the four nozzles. Since these range of intensities largely fall in the mixing layer of the jet they have been termed the Jet Shear Layer. However, it should be borne in mind that the acetone PLIF intensity is also affected strongly by, pressure temperature and quenching effects near regions of shock influence. Hence in certain cases (for example in small pockets that are visible at the jet center in Type $\mathrm{C}$ images) this fact has to be considered while interpreting the images. However, the jet shear layers are emphasized so distinctly in all the cases that there is no unambiguity in the inferences. Further, it should be understood that the thickness observed here are visual thickness and actual quantitative estimates are computed only for the entire jet width which includes the core and shear layer. Clearly, the jet shear layer thickness is more in the case of Type $\mathrm{C}$ and Type D nozzles than in Type A or Type B. This indicates significant enhancement of mixing in Type $\mathrm{C}$ and Type D nozzles. The thickness of shear layer is higher in case of Type D than in Type C. The overall jet width can now be visualized clearly in the recomposed images wherein the jet shear layer is emphasized by applying a higher gain (Section 3.1.1) as shown in Figure 6c. The qualitative trends of mixing enhancement in complex nozzles in comparison to the reference round nozzle Type A has been discussed. From the recomposed images the jet boundary including the spread of the mixing layer is clearly visible. Thus, this jet boundary width is extracted from the recomposed images and is plotted in Figure 7. The plots correspond to only those locations that show maximum increase of jet width in the cases of nozzles Type $\mathrm{C}$ and 
Type D respectively.

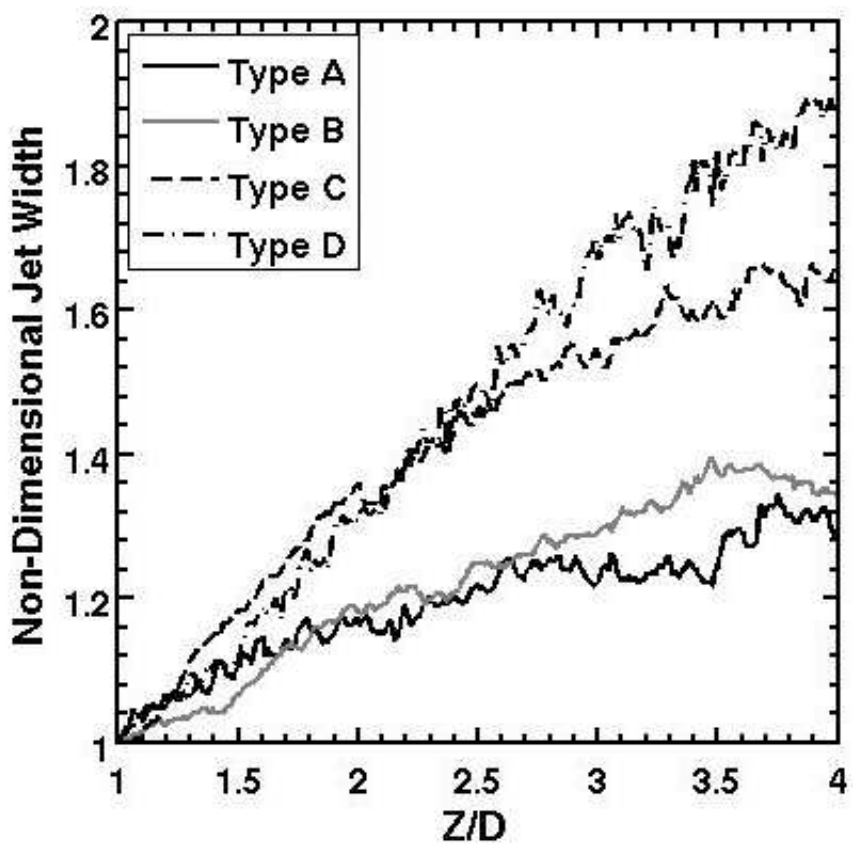

Figure 7: Variaton of Non-Dimensional Jet Width with $\mathrm{Z}$ for four different nozzles from $1 \mathrm{D}$ to $4 \mathrm{D}$ along $\mathrm{Z}$ axis.

. The jet width is non-dimensionalized by the jet width at the start of the analysis window (termed $\mathrm{W}$ in further discussions) to render the plot such that the rate of increase of jet width is clearly evident. From the plots it is clear that the growth rate of the jet width is much higher in case of nozzles Type $\mathrm{C}$ and Type D compared to Type A and Type B. Type D shows the largest increase in growth rate of jet width in comparison to Type C. The average slope of the lines (W vs $\mathrm{Z})$ is evaluated by taking a linear fit and computing its slope (dW/dZ) [1]. The increase in growth rate when using complex nozzle is determined by taking the ratio $\left(\frac{d W / d Z}{d W / d Z_{\text {Type }}}\right)$. Table 2 tabulates the average values of growth rate of jet width as evaluated from the PLIF images. The conical nozzle is taken as the reference and then it is seen that the bevel nozzle Type B improves mixing and jet growth by a moderate $138 \%$. The increase is more significant in the case of Type C (222\%) and Type D (430\%) nozzles. Thus, the ESTS lobed nozzle is shown to increase the growth rate to about 4.3 times the round nozzles growth 


\begin{tabular}{|l|c|c|c|c|}
\hline Nozzle Type & Type A & Type B & Type C & Type D \\
\hline $\begin{array}{l}\text { Growth rate } \\
\mathrm{dW} / \mathrm{dZ}\end{array}$ & 0.072 & 0.099 & 0.159 & 0.308 \\
\hline$\%$ Increase & - & $138 \%$ & $222 \%$ & $430 \%$ \\
\hline
\end{tabular}

Table 2: Table of average growth rate of jet width vs $\mathrm{Z}$

rate, almost twice higher than the chevron nozzle (2.22 times).

\subsection{Cross-sectional PLIF}

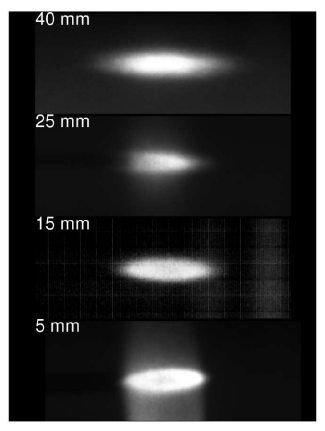

a) Type A

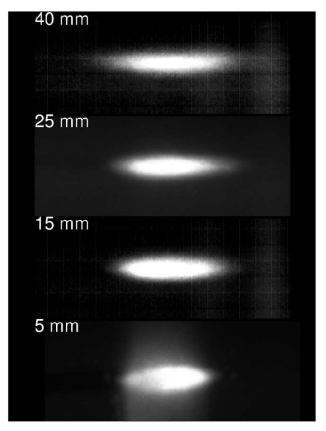

b) Type B

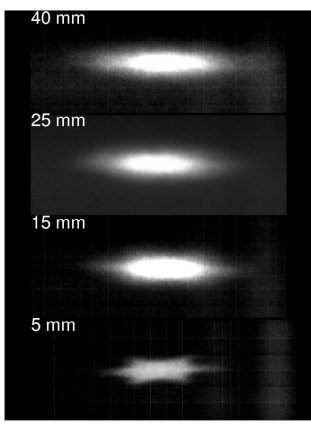

c) Type C

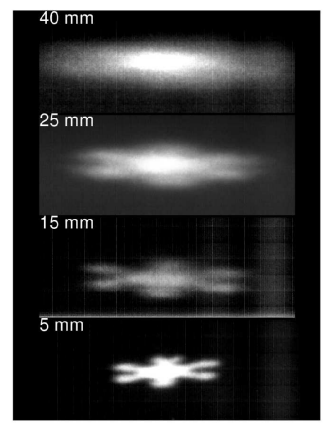

d) Type D

Figure 8: Comparison of average PLIF images takes at cross-sectional planes at different Z locations.

The comparison of average PLIF images at different cross-sectional planes along the $\mathrm{Z}$ direction is given in Figure 8. The planes are located at distances $5,15,25$ and $40 \mathrm{~mm}$; which in non-dimensional units are at $\mathrm{D}, 3 \mathrm{D}, 5 \mathrm{D}$ and $8 \mathrm{D}$ respectively. While the images have been stacked vertically according to the $\mathrm{Z}$ location, it should be noted that the camera viewing angle is not the same for each of the $\mathrm{Z}$ locations. This is particularly visible in the comparison of images taken at $\mathrm{Z}=25 \mathrm{~mm}$ and $\mathrm{Z}=15 \mathrm{~mm}$. There is expected to be a much greater spread of the jet at $Z=25 \mathrm{~mm}$, than at $\mathrm{Z}=15 \mathrm{~mm}$, but the change in viewing angle 
makes the difference appear less apparent. At a particular Z location the camera viewing angle is maintained constant for the different nozzle cases. Thus, the comparisons regarding the spread of the jet at particular Z location among the four nozzles is valid.

. Type A is a round supersonic jet, which appears elliptical due to the perspective. As evidenced in the streamwise visualizations, the jet from Type A does not spread as much and more or less maintains its shape. In effect, the jet from Type B is not very different from Type A, the cross section is circular and the asymmetry is evident only in the streamwise planes. The features of both Type $\mathrm{C}$ and Type D nozzle that have complex nozzle geometry are interesting. The jet structure in the cross-sectional planes close to the nozzle $\mathrm{Z}=15 \mathrm{~mm}$ resembles the exit shapes of the nozzle. Since the chevrons are triangular shaped with the tips at the exit and wide gaps between the tips, the shape in PLIF images resembles six fingered star shape, with the core flow extending to the tips while the ambient flow protrudes into the center at the gaps. Streamwise vorticity is generated at the chevrons which interact with each other and the ambient enhancing mixing. This interaction results in smoothening of the star shape which becomes nearly elliptical at $\mathrm{Z}=25 \mathrm{~mm}$ and at $\mathrm{Z}=40 \mathrm{~mm}$ it is completely elliptical. The six lobed structure is visible in Type $\mathrm{D}$ as well, the core flow extending at the lobe crests and the penetration of ambient at the lobe tips. Similar to Type $\mathrm{C}$ the action of vortices besides spreading the jet widely also makes the shape more smooth. But even at $Z=25 \mathrm{~mm}$ jet from Type $\mathrm{D}$ maintains its distinct shape and it is lost completely at $\mathrm{Z}=40 \mathrm{~mm}$. These effects where in the ambient fluid is brought close to the jet center by the tips of lobed nozzle or the gaps of the chevron nozzle is called the pinching effect [1]. Further, this enhances the shear perimeter of the nozzle which is another reason for increased entrainment and mixing. Clearly at every $\mathrm{Z}$ location the spread of the jet is widest in Type $\mathrm{D}$ followed by Type $\mathrm{C}$. The spread of the jet can be estimated by calculating the area of pixels which contain intensities corresponding to the spread of acetone tracers as explained in Section 3.1.2. The jet cross sectional area is expressed in pixel units and is compared only at particular $\mathrm{Z}$ locations where the area transformation on camera viewing is maintained constant. From Table 3, which tabulates the cross-sectional areas for the jets at $\mathrm{Z}=15 \mathrm{~mm}$ and $\mathrm{Z}=40 \mathrm{~mm}$, it is very evident that Type $\mathrm{C}$ and 


\begin{tabular}{|l|c|c|c|c|}
\hline Nozzle Type & Type A & Type B & Type C & Type D \\
\hline \multicolumn{5}{|c|}{$\mathrm{Z}=15 \mathrm{~mm}$} \\
\hline Area (A) & $2.94 \times 10^{4}$ & $3.93 \times 10^{4}$ & $4.73 \times 10^{4}$ & $5.85 \times 10^{4}$ \\
\hline $\mathrm{A} / \mathrm{A}_{\text {Type }}$ & 1.00 & 1.34 & 1.61 & 1.99 \\
\hline \multicolumn{4}{|c|}{$\mathrm{Z}=40 \mathrm{~mm}$} & \\
\hline Area $(\mathrm{A})$ & $5.40 \times 10^{4}$ & $4.60 \times 10^{4}$ & $6.04 \times 10^{4}$ & $1.24 \times 10^{5}$ \\
\hline $\mathrm{A} / \mathrm{A}_{\text {Type }}$ & 1.00 & 0.85 & 1.12 & 2.29 \\
\hline
\end{tabular}

Table 3: Table of cross sectional areas (in pixel units) evaluated from cross-sectional PLIF images for the four nozzles.

Type $\mathrm{D}$ generate a greater spread. At the farthest $\mathrm{Z}$ location where the shape is elliptical for all the nozzles, Type D has 2.29 times the area compared to Type A indicating significant enhancement of mixing, about double compared to Type C (1.12).

\subsection{Pitot pressure measurements}

Pitot pressure is measured at discrete locations along the centerline of the jet from $4 \mathrm{D}$ to $20 \mathrm{D}$ using a pitot tube of $0.9 \mathrm{~mm}$ diameter. The ensemble averaged pitot measurements at each location for different nozzles is plotted in Figure 9. Also plotted are the corresponding pitot pressures obtained from CFD computations. The pitot pressures are normalized by the stagnation pressure measured at the stagnation chamber. The deviation of experimental data for multiple runs at the same conditions is within $2 \%$ for all the cases which is well within the expected uncertainty of $6.5 \%$ indicating the reliability of the measured values. The trends of centerline pitot measurements reveal the changes to centerline Mach number. The centerline Mach number changes due to the presence of shockexpansion features and the progress of mixing with the ambient. From the PLIF 


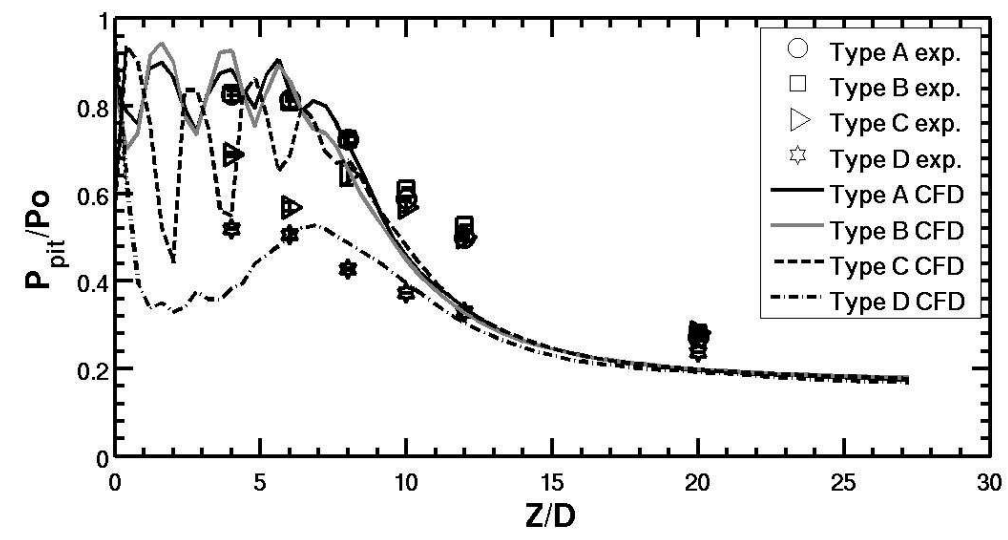

Figure 9: Plots of pitot pressure obtained from experiment and CFD for the four different nozzles.

images it is clear that the shocks are generally weak for Type A and Type B. Further, no significant differences are found in the jet spread trends, hence both Type A and Type B show similar pitot pressure trends. Type C shows a larger reduction in pitot pressures within the near field of the jet due to both enhanced mixing as well as the presence of shocks. After 10D, however, the pitot pressure trends of Type $\mathrm{C}$ approach that of Type A and Type B. The cross sectional PLIF images also show the loss of near field character quite quickly in the case of Type C. Type D shows the largest decrease in pitot pressure which can be due to the coupled effect of relatively high mixing rate as evidenced by the PLIF images and also due to the fact that a strong Mach disk is observed at the first shock cell. The flow regains a part of the supersonic character downstream of the Mach disk, but subsonic pockets are to be expected. The cross sectional PLIF images also show that Type D maintains its relatively high mixing rates and complex shapes farther than Type C. Therefore in the pitot trends Type D joins the curves of other nozzles at the last. These inferences on the variations of centerline Mach number are borne out by the Mach number contours from the CFD simulations which are described in Section 5.4. 
. The validity of the numerical simulations can be easily evaluated from the comparison of the pitot pressures obtained from experiment and CFD. Some factors which have to be considered when comparing the results are that the CFD results do not include the effect of the pitot probe which is an intrusive measurement technique. It is to minimize the effects of the intrusion that experimental pitot measurements are conducted from 4D onwards. Pitot tube in a supersonic flow will produce a typical detached bow shock structure ahead of it which will affect the flow. The effects of pitot are more complicated in downstream regions where the flow may contain pockets of supersonic and subsonic flow. Studies have shown that nearly $10 \%$ difference can be caused due to the pitot in supersonic dominated regions of the round jet and the difference is expected to increase in mixed regions of the jet [39]. The second factor is the finite area of sensing that is offered by the pitot, to account for this fact an average of pitot pressure is taken over an area corresponding to the piot tube diameter in the CFD results. Even if this is considered it is considerably different from the exact experimental measurement. The third factor is the fact that the mixing rate is highly dependent on the turbulent nature of the flow and the emerging vorticies, which are not exactly computed but are modeled through a turbulence model. The differences in prediction of mixing rate also affects the prediction of pitot measurements from CFD. The pitot pressure is computed from the CFD results by using the Rayliegh pitot equation wherever the flow is supersonic and using the isentropic pitot equation in cases of subsonic flow [34].

. Even if all these factors are considered, it is seen that in the supersonic regions $(\mathrm{Z}<10 \mathrm{D}$ in the plot) of the flow for nozzles Type A and Type $\mathrm{B}$ that do not contain strong shocks, the difference between CFD and experiment is $6 \%$ which is well within the range of difference reported earlier [39]. The difference does increase downstream to a maximum of $30 \%$ at $12 \mathrm{D}$. The CFD pitot profile closely follows the experimental curve despite such local differences. In Type $\mathrm{C}$ except at $6 \mathrm{D}$ where the pitot is located exactly close to the shock, the differences between CFD and experiments are the same as Type A. Similarly in Type D where there is a Mach disk present, the difference is high at $4 \mathrm{D}$, but thereafter the CFD and experimental curves are close to each other. The CFD results clearly depict the experimental observations such as pitot pressures are less in the near field for 
Type C and much reduced in Type D. Further, they also show that the pitot pressure profile of Type $\mathrm{C}$ approaches that of Type A earlier than Type D. Far downstream all pitot pressure profiles merge together. Considering all these facts it can be concluded that the CFD results very well represent the flow features and trends as observed in the experiments. The results from CFD can be used to compare aspects of the complex nozzles such as vorticity distributions.

\subsection{Numerical Results}

Figure 10a shows the Mach number contours for streamwise planes at the same locations as the PLIF images were taken. The images extend to about $12.5 \mathrm{D}$, which is longer than the PLIF images. Clearly, the flow features within 6D correspond to similar average PLIF images described in Section 5.1. Type A nozzle does not contain strong shock structures and similarly the asymmetry of Type B is also clearly visible. The shock structure undergoes a zig-zag and the flow sightly bends towards the shorter end. The shock structure that begins upstream of Type $\mathrm{C}$ exit is clearly seen in this figure, where as only the end of this first shock cell was visible in PLIF images. The shock ends in Mach disk of very small diameter and the subsonic pocket results in a shear layer which can also be seen affecting the flow features downstream of this location. the three dimensional nature of the flow field is evident in the differences between the flow features in the sections taken at gaps and tips of Type C nozzle. Similarly, the Mach disk in Type D is also evident. A region of subsonic flow is also visible downstream and so are the differences of flow features at lobe crest and tips. A significant point to notice is that the highest Mach number encountered gets progressively higher from Type A to Type D. The highest Mach number is 2.50 for Type C and 3.0 for Type D respectively. This is due to the fact that variations in the cross sectional area of the flow at the lobe tips and lobe crests in Type D, and the gaps and tips of the chevrons in Type $\mathrm{C}$ cause local underexpansion and overexpansion. This is also the reason for the observation of oblique shocks from within the nozzle in Type C.

. The Mach number distributions across the X-Y planes at the same locations as the PLIF images are shown in Figure 10b. The similarity between flow features as visible in PLIF images and CFD results are evident. CFD results are taken at orthogonal slices and hence do not exhibit the perspective distortion in PLIF 


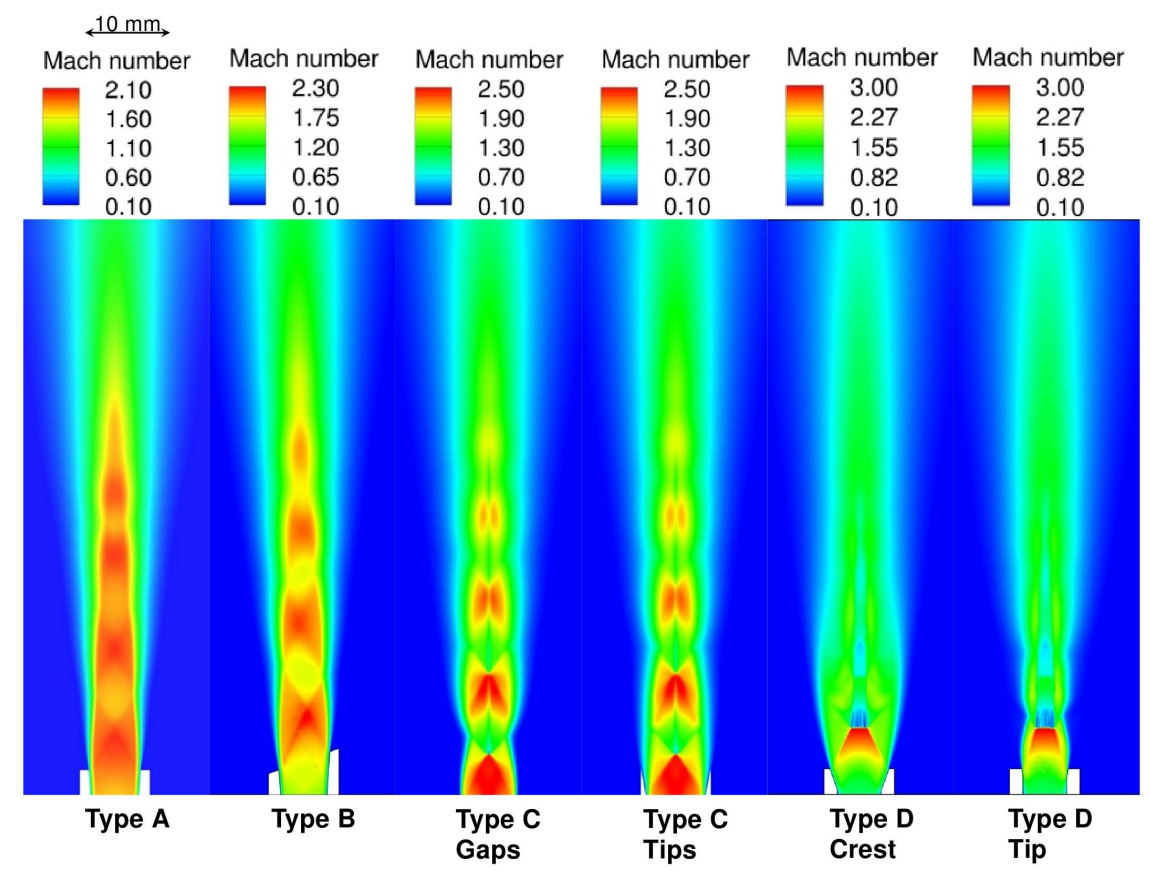

a) Comparison of Mach number contours along streamwise planes at the same locations as PLIF images

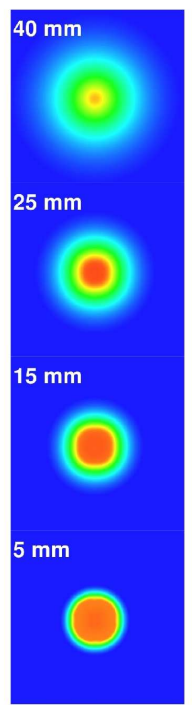

Type A

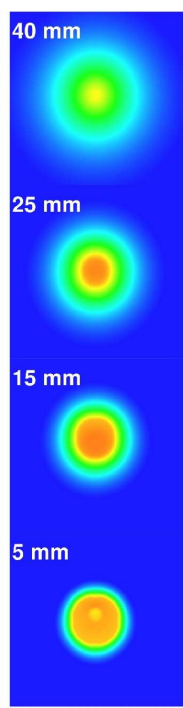

Type B

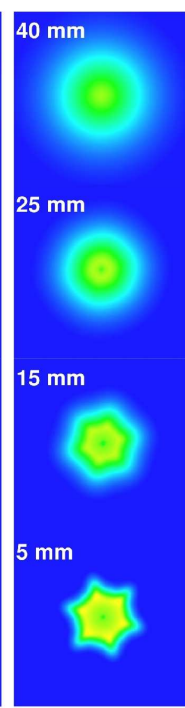

Type C

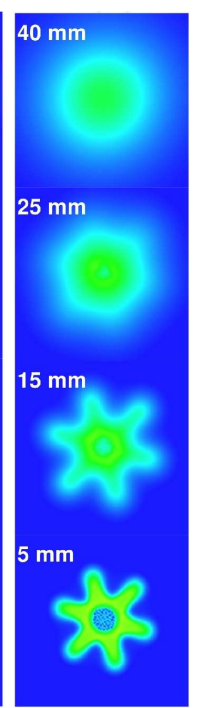

Type D

b) Comparison of Mach number contours along cross-sectional planes at the same locations as PLIF images

Figure 10: Comparison of CFD results along streamwise and cross-sectional planes at the same locations as PLIF images were captured. 
images. The Mach contours of Type A nozzle are concentric, while in Type B the circles corresponding to the jet core are seem offset slightly particularly at $\mathrm{Z}=5 \mathrm{~mm}$ plane. The overall character of Type $\mathrm{B}$ is not very different from Type A. Type $\mathrm{C}$ shows the 6 pointed star shape, with the points located at the tips. Downstream the shape loses sharpness and by $25 \mathrm{~mm}$ it shows circularity as observed in the PLIF images. The lobed structure of Type D shows a dominant subsonic region at the center due to the Mach disk. Similar to observations in PLIF images Type D loses its lobe structure later than Type C at $40 \mathrm{~mm}$. Clearly, the observations from Mach contours from CFD confirm the inferences of flow structure from PLIF images. The most important aspect that results in enhanced mixing from the complex nozzles is the production of streamwise vortices and their interaction, besides other factors such as increase in shear perimeter and pinching effects [1]. A comparison of streamwise vorticity magnitudes can be now evaluated from the CFD images. The vorticity in the streamwise direction

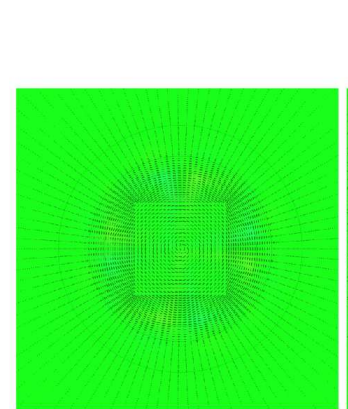

a) Type A

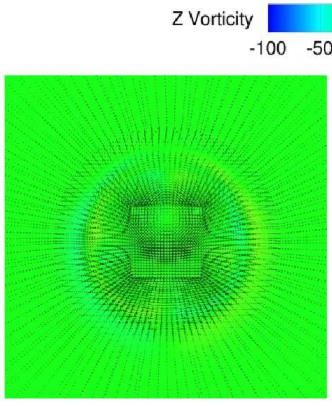

b) Type B
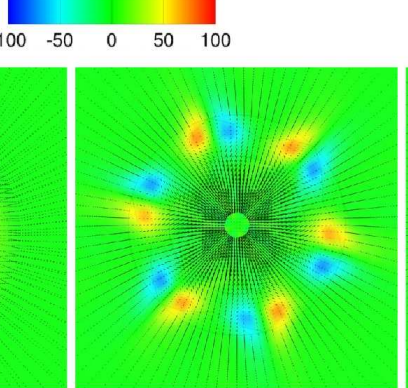

c) Type C

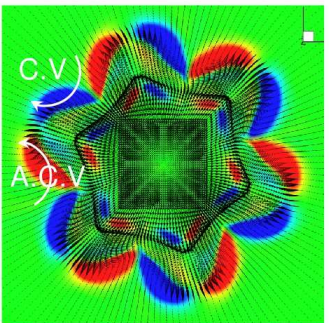

d) Type D

Figure 11: Comparison of contours of vorticity in $\mathrm{Z}$ direction along with velocity vectors calculated from the $C F D$ results at $\mathrm{Z}=2 \mathrm{~mm}$.

generated from the nozzles is depicted in Figure 11 close to the nozzle exit at $\mathrm{Z}=2$ $\mathrm{mm}$ such that it is well before the first shock cell that contains the Mach disk. The vorticity present along the shear layers of Type A and Type B are small, Type B showing slightly higher magnitude compared to Type A, but small hence not distinguishable. The point of interest is the comparison of the vorticity and their influence in Type $\mathrm{C}$ and Type $\mathrm{D}$ nozzles. Both nozzles produce significant streamwise vorticity evidenced by the red and blue regions indicating the counterrotating character of the vortices, around every chevron tip or lobe respectively. 
The vorticity dominant regions are small and clustered at the pointed ends of the chevron tips, consequently the cross sectional velocity vectors are not high in magnitude and are clustered. On the other hand, in Type D the vorticity is not only high it is wide spread as well, generating significant velocity vectors around the lobes. The directions of the velocity vectors indicate the clockwise (C.V.) and anti-clockwise (A.C.V) nature of the vortices marked in the images. It is important to notice the directions of the velocity vectors which to the ambient flow are directed such that more ambient flow is injected into the core of the jet at the lobe tip and the jet fluid is pushed out significantly at the lobe crests. This observation confirms the conjectures put forward in the explanations by Rao and Jagadeesh [1] regarding the character of the vortices generated by the ESTS lobed nozzle. The mechanism for vorticity generation in chevron and tabbed geometries is well explained in the previous studies by Zaman et.al., Reeder and Samimy [11, 13, 12] and Hu et.al. [14]. Though the mechanism in the case of ESTS lobed nozzles is advanced by Rao and Jagadeesh [1], it has not been substantiated due to the lack of experimental or numerical observations. From the current CFD simulations the mechanism for vorticity production can be readily verified. The zoomed image focusing on the interface of a pair of lobe crest and tip with pressure contours and velocity vectors at $\mathrm{Z}=2 \mathrm{~mm}$ is shown in Figure 12. The surrounding ambient fluid is at ambient pressure. It is easily seen that if one sweeps along azimuthally at a constant radius the pressure decreases towards the lobe crest. This is due to local azimuthal variations of Mach number. It is important to understand that in the lobed nozzle the lobe shapes proceed from the throat to exit of the nozzle. Hence, the Mach number is higher at lobe crests (as they expand more and results in lower pressures) than at lobe tips (which penetrate the jet and lead to relatively higher pressure), thereby generating this pressure gradient. The flow then responds to this pressure gradient as is evident from the directions of the velocity vectors which move from the lobe tip to lobe crest. Thus, the ambient fluid is pulled into the core at the lobe tips while the jet fluid is significantly expelled at the lobe crests. This causes significant streamwise vortices of large area of influence. This generation of large streamwise vortices is the key mechanism for mixing enhancement. 


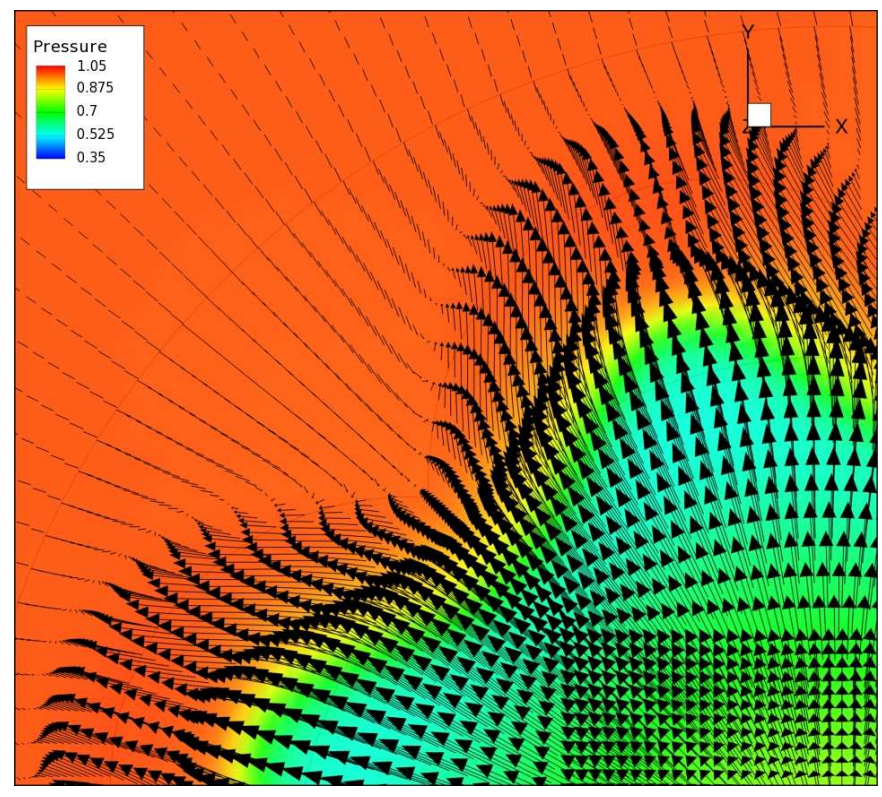

Figure 12: Pressure contours and velocity vectors at the interface of a pair of lobe crest and tip of Type D nozzle.

\subsection{A discussion on complex nozzles}

Consolidating all the results, it is evident that Type $\mathrm{C}$ and Type D nozzles enhance mixing significantly compared to Type B. The dramatic changes brought about by Type $\mathrm{C}$ and Type $\mathrm{D}$ show up in a marked decrease of pitot pressures in the near field, more for Type D than for Type C. Both Type C and Type D show stronger shocks which can lead to higher stagnation pressure losses. The shock structure is three dimensional, affected by the azimuthal variations of Mach number. From quantitative comparisons of the jet width growth rate it is shown that the ESTS lobed nozzle (Type D) increases mixing to $430 \%$, about twice as much as Chevron nozzle $220 \%$ (Type C). One of the primary reasons for this difference can be attributed to the effectiveness of vorticity production. The ESTS lobed nozzle generates a significant azimuthal Mach number variation from the throat to the exit of the nozzle leading to development of large vortices on exit. The chevrons, on the other hand have a flow structure within the nozzle which is same as the round nozzle for most of the nozzle until they encounter the chevrons close to the exit. Thus, the influence of the chevrons are restricted 
near the exit of the nozzle hence they do not produce as much azimuthal variation of Mach number and the vortices are rather clustered close to the chevron tips. There are other factors to be considered before generalizing this result. It is observed that the first shock cell arises upstream in Type C nozzle very close to the start of the chevrons. Shock-boundary layer interaction at this location can significantly reduce the effect of chevrons [17]. The chevrons can be bent into the nozzle (penetration angle), but they lead to significant stagnation pressure loss $[17,15]$ So the penetration angle has usually been kept small. No specific geometrical penetration is given in this study. However, the expanding supersonic flow may undergo a small degree of local penetration. Similarly, the Mach disk observed in the case of Type D nozzle is due to local underexpansion and variation of azimuthal Mach number. Both chevron and ESTS lobed nozzle have a number of geometrical parameters that can be optimized and such a study has to be undertaken. If previous studies into the use of these nozzles in supersonic ejectors are considered, then also the observations show that ESTS lobed nozzle (maximum increase of entrainment 30\%) [1] performs better than chevron nozzle (maximum increase 21\%)[2]. The application to supersonic mixing in ejectors and air-breathing engines demand significant enhancement of mixing rates where complex nozzles can be used effectively. The advantage with ESTS lobe nozzle is that it generates significant streamwise vortices and is easy to design. Complex shock structure and high penetration that result in severe stagnation pressure losses from deeply convoluted lobed nozzles is minimized to considerable extent in ESTS lobed nozzle.

\section{Conclusion}

Comparative studies are conducted among four supersonic nozzles of Mach number 1.80, at nozzle pressure ratio of 6 . A conical supersonic nozzle with round exit of $5 \mathrm{~mm}$ throat diameter is taken as the reference nozzle. For the rest of the nozzles the throat diameter remains the same and modifications are made to the exit geometry. This results in an beveled nozzle, a chevron nozzle with six chevrons and an ESTS lobe nozzle of six lobes. Experimental studies use acetone PLIF technique to understand the mixing process of the supersonic jet and centerline pitot measurements. Numerical studies are conducted using FASTAR 
by solving three dimensional RANS equations using the Spalart-Allmara's turbulence model on a mesh consisting of 8 million hexahedral cells. A comparison of experimental and numerical pitot pressures show that within the supersonic regions the agreement is within $6 \%$ while the largest difference encountered is $30 \%$ in regions of mixed flow. The intrusive nature of pitot measurements is not considered while comparing the CFD and experimental results. Despite this fact, the trends of pressure profiles are well predicted by CFD, thus validating the numerical procedure. The acetone PLIF images are appropriately processed to emphasize the mixing layers of the jets whereby the correct extent of jet spread can be made visible. The acetone PLIF images reveal slight changes to the symmetry of the flow in beveled nozzle but moderate change in mixing characteristics. Both chevron and ESTS lobed nozzle show significant changes including stronger shocks, significant three dimensionality of the flow and high rates of mixing. Mixing enhancement is characterized by the growth rate of the jet width in streamwise visualizations and the area of jet spread in cross-sectional visualizations. The enhancement of mixing is found to be $138 \%$ in beveled nozzle, $222 \%$ in chevron nozzle and $430 \%$ in ESTS lobed nozzle. The CFD Mach number contours agree well with the flow picture that emerges from PLIF visualizations. Vorticity contours show counter rotating vortices of significant strength in chevron and ESTS lobed nozzle. The vortices are clustered near the tip of chevrons in the chevron nozzle. ESTS lobed nozzle where there is a strong azimuthal variation of Mach number from the throat to exit of the nozzle is shown to effectively generate large scale streamwise vortices. However several factors are yet to be considered such as optimization of ESTS lobe and chevron geometries before generalizing the conclusion regarding the effectiveness of one over the other. The numerical results also verify the mechanism of streamwise vorticity production in ESTS lobed nozzles for the first time. The lobes produce favorable pressure gradients from the lobe tip to the lobe crest causing the flow to roll up along the lobe periphery from lobe tip to lobe crest thus generating large streamwise vortices. This also results in the pinching effect wherein the ambient fluid penetrates into the core at the lobe tips while the jet fluid is expelled at the lobe crests. To emphasize the significant conclusions of the study:

- The beveled nozzle shows moderate enhancement of mixing (138\%), while the chevron nozzle shows $222 \%$ and ESTS lobed nozzle $430 \%$. 
- ESTS lobed nozzle shows about double the mixing enhancement compared to chevron nozzles.

- Streamwise vortices are shown to be more effectively produced, are of significant strength and range of influence in the ESTS lobed nozzle compared to chevron nozzle.

- The mechanism of vorticity production in the ESTS lobed nozzle through the generation of a favorable pressure gradient along the lobe tips to crest that cause the flow to roll up is confirmed from CFD results.

Studies into optimization of geometrical parameters for most efficient mixing enhancement in complex nozzles need to be conducted.

\section{Acknowledgements}

The authors are grateful to JAXA, Japan for allowing the use of CFD code FASTAR and to the members of Propulsion Engineering Laboratory, Muroran Institute of Technology for their help in the work.

\section{References}

[1] Rao, S.M., Jagadeesh, G.. Novel supersonic nozzles for mixing enhancement in supersonic ejectors. Applied Thermal Engineering 2014;71:62-71.

[2] Kong, F., J, Y., Setoguchi, T., Kim, H.D.. Numerical analysis of chevron nozzle effects on performance of the supersonic ejector-diffuser system. Journal of Thermal Science 2013;22(5):459-466.

[3] Srikrishanan, A., Kurien, J., Sriramlu, V.. Experimental study on mixing by petal nozzle in supersonic flow. Journal of Propulsion and Power 1996;12(1):165-169.

[4] Timan, T., Paterson, R., (Jr.), W.P.. Supersonic nozzle mixer ejector. Journal of Propulsion and Power 1992;8(2):513-519.

[5] Papamoschou, D., Roshko, A.. The compressible turbulent shear layer: an experimental study. Journal of Fluid Mechanics 1988;197:453-477.

[6] Clemens, N.T., Mungal, M.G.. Large-scale structure and entrainment in the supersonic mixing layer. Journal Of Fluid Mechanics 1995;284:177-216.

[7] Gutmark, E., Schadow, K., Yu, K.. Mixing enhancement in supersonic free shear flows. Annual Review of Fluid Mechanics 1995;27:375-417.

[8] Narayanan, A., Damodaran, K.. Supersonic ejector characteristics using a petal nozzle. Journal of Propulsion and Power 1994;10(5):742-743. 
[9] Srikrishnan, A.R., Kurian, J., Sriramulu, V.. An experimental investigation of thermal mixing and combustion in supersonic flows. Combustion And Flame 1996;107:464-474.

[10] Opgenorth, M.J., Sederstrom, D., McDermott, W., Lengsfeld, C.S.. Maximizing pressure recovery using lobed nozzles in a supersonic ejector. Applied Thermal Engineering 2012;37:396-402.

[11] Zaman, K., Reeder, M.F., Mungal, M.G.. Control of an axisymmetric jet using vortex generators. Physics of Fluids 1994;6(2):778-793.

[12] Reeder, M.F., Samimy, M.. The evolution of a jet with vortex-generating tabs: real-time visualization and quantitative measurements. Journal of Fluid Mechanics 1996;311:73-118.

[13] Zaman, K.. Spreading characteristics of compressible jets from nozzles of various geometries. Journal of Fluid Mechanics 1999;383:197-228.

[14] Hu, H., Saga, T., Kobayashi, T., Taniguchi, N.. Research on the vortical and turbulent structures in the lobed jet flow using laser induced fluorescence and particle image velocimetry techniques. Measurement Science and Technology 2000;19:698-711.

[15] Zaman, K., Bridges, J., Huff, D.. Evolution from 'tabs' to 'chevron technology a review. In: Proceedings of the 13th Asian Congress of Fluid Mechanics, Dhaka, Bangladesh. 2010,

[16] Kuo, C.W., Veltin, J., McLaughlin, D.K.. Advanced acoustic assessment of small-scale military-style nozzles with chevrons, aiaa 2010-3923. In: 16th AIAA/CEAS Aeroacoustics Conference. 2010,

[17] Kurbatskii, K.A.. Comparison of rans turbulence models in numerical prediction of chevron nozzle jet flows, aiaa 2009-499. In: 47th AIAA Aerospace Sciences Meeting Including The New Horizons Forum and Aerospace Exposition, Orlando, Florida. 2009,

[18] Tide, P.S., Srinivasan, K.. Aeroacoustic studies of beveled and asymmetric-chevron nozzles, aiaa 2009-3404. In: 15th AIAA/CEAS Aeroacoustics Conference (30th AIAA Aeroacoustics Conference), Miami, Florida. 2009,.

[19] Schlinker, R., Simonich, J., Shannon, D., Reba, R., Colonius, T., Gudmundsson, K., et al. Supersonic jet noise from round and chevron nozzles: Experimental studies, aiaa 2009-3257. In: 15th AIAA/CEAS Aeroacoustics Conference (30th AIAA Aeroacoustics Conference), Miami, Florida. 2009,.

[20] Viswanathan, K., Spalart, P.R., Czech, M.J., Garbaruk, A., Shur, M.. Tailored nozzles for jet plume control and noise reduction. AIAA Journal 2012;50(10):375-417.

[21] Webster, D.R., Longmire, E.K.. Vortex dynamics in jets from inclined nozzles. Physics of Fluids 1997;9(3):655-666.

[22] Samitha, Z.A., Davis, D., Balachandran, P.. Computational study on supersonic mixing using clover nozzle, aiaa 2009-30. In: 47th AIAA Aerospace Sciences Meeting Including The New Horizons Forum and Aerospace Exposition, Orlando, Florida. 2009,

[23] Yang, X., Long, X., Yao, X.. Numerical investigation on the mixing process in a steam ejector with different nozzle structures. International Journal of Thermal Sciences 2012;56:95-106.

[24] Longmire, E.K., Eaton, J.K., Elkins, C.J.. Control of jet structure by crown shaped nozzles. AIAA Journal 1992;30(2):505-512.

[25] Shelar, V.M., Rao, S.M.V., Hegde, G.M., Umesh, G., Jagadeesh, G., Reddy, K.. Acetone 
planar laser-induced fluorescence for supersonic flow visualization in air and nitrogen jet. International Journal of Mechanical and Materials Engineering 2014;9(28).

[26] Hatanaka, K., Saito, T., Nakamura, Y., Suzuki, Y., Koyaguchi, T.. Flow visualization of supersonic free jet utilizing acetone plif. Visualization of Mechanical Processes 2012;2(1).

[27] Reid, J.Z., Lynch, K.P., Thurow, B.S.. Density measurements of a turbulent wake using acetone planar laser-induced fluorescence. AIAA Journal 2013;51(4):829-839.

[28] A. Lozano, B.Y., Hanson, R.K.. Acetone: a tracer for concentration measurements in gaseous flows by planar laser-induced fluorescence. Experiments in Fluids 1992;13:369-376.

[29] Handa, T., Masuda, M., Kashitani, M., Yamaguchi, Y.. Measurement of number densities in supersonic flows using a method based on laser-induced acetone fluorescence. Experiments in Fluids 2011;50:1685-1694.

[30] Thurber, M., Hanson, R.. Pressure and composition dependences of acetone laser-induced fluorescence with excitation at 248, 266, and 308 nm. Applied Physics B 1999;69:229-240.

[31] van Cruyningen, I., Lozano, A., Hanson, R.K.. Quantitative imaging of concentration by planar laser-induced fluorescence. Experiments in Fluids 1990;10:41-49.

[32] Bryant, R.A., Donbar, J.M., Driscoll, J.F.. Acetone laser induced fluorescence for low pressure/low temperature flow visualization. Experiments in Fluids 2000;28:471-476.

[33] Thurber, M.C., Grisch, F., Kirby, B.J., Votsmeier, M., Hanson, R.K.. Measurements and modeling of acetone laser-induced fluorescence with implications for temperature-imaging diagnostics. Applied Optics 1998;37(21):471-476.

[34] Tropea, C., Yarin, A.L., Foss, J.F.. Springer Handbook of Experimental Fluid Mechanics. 2007.

[35] Hashimoto, A., Murakami, K., Aoyama, T., Ishiko, K., Hishida, M., Sakashita, M., et al. Toward the fastest unstructured cfd code fastar, aiaa 2012-1075. In: 50th AIAA Aerospace Sciences Meeting including the New Horizons Forum and Aerospace Exposition, Nashville, Tennessee, 09 - 12 January. 2012,

[36] Hashimoto, A., Murayama, M., Yamamoto, K., Aoyama, T., Tanaka, K.. Turbulent flow solver validation of fastar and upacs, aiaa 2014-0240. In: 52nd Aerospace Sciences Meeting, National Harbor, Maryland, 13-17 January. 2014,.

[37] Argyropoulos, C., Markatos, N.. Recent advances on numerical modelling of turbulent flows. Applied Mathematical Modelling 2014;39(2):693-732.

[38] Deck, S., Duveau, P., dEspiney, P., Guillen, P.. Development and application of spalartallmaras one equation turbulence model to three-dimensional supersonic complex configurations. Aerospace Science and Technology 2002;6:171-183.

[39] XiWen, Z., PengFei, H., ZhaoHui, Y.. The measurement error analysis when a pitot probe is used in supersonic air flow. Science China, Physics, Mechanics and Astronomy 2011;54(4):690-696.

[40] Kim, Y., Nakamura, Y.. Effect of nozzle lip shape on screech tone in a supersonic jet. Transactions of Japanese Society for Aeronautical and Space Sciences 2008;51(171):1-7. 